\title{
In-Situ UV-Photopolymerized PVA-g-GMA Hydrogels for Biomedical Applications: I. Synthesis, Characterizations and Grafting Optimization
}

\author{
Elbadawy A. Kamoun", Ahmed M. Omer', Sherine N. Khattab², Heba M. Ahmed', Ali A. Elbardan² \\ 'Polymeric Materials Research Dep., Advanced Technology and New Materials Research Institute, City for Scientific Research and Technological Applica- \\ tions (SRTA-City), New Borg Al-Arab 21934, Alexandria, Egypt. \\ ${ }^{2}$ Chemistry Department, Faculty of Science, Alexandria University, P.O. Box 426, Ibrahimia, Alexandria 21321, Egypt.
}

\begin{tabular}{l}
\hline ARTICLE INFO \\
\hline Article history: \\
Received on: 27/08/2017 \\
Accepted on: 18/11/2017 \\
Available online: 28/01/2018 \\
\hline Key words: \\
Poly (vinyl alcohol), Glyc- \\
idyl methacrylate, hydrogels, \\
photocrosslinking, biomedi- \\
cal applications
\end{tabular}

\begin{abstract}
This work explores the synthesis and grafting optimization of PVA-g-GMA hydrogels. The grafting of poly (vinyl alcohol) (PVA) with glycidyl methacrylate (GMA) was conducted by the trans-esterification reaction via introducing methacryloyl groups into PVA chains and glycidol was formed as by-product. The grafting reaction conditions of PVA-g-GMA e.g. GMA concentrations and reaction temperature were optimized. The kinetic parameters e.g. grafting efficiency (GE) and grafting percentage (GP\%) were calculated to optimize the grafting reaction, while yield (\%) was determined to monitor the hydrogels formation. The instrumental characterizations e.g. ${ }^{1} \mathrm{H}-\mathrm{NMR}$, FTIR, SEM and TGA/DSC, were investigated for verifying the grafting reaction. The UV-photopolymerization was used for photocrosslinking the water-soluble PVA-g-GMA using Irgacure $2959\left(\mathrm{I}_{2959}\right)$ as a photoinitiator. Results revealed that the grafting reaction dramatically increased with increase of both GMA concentration until $0.15 \mathrm{M}$ and reaction temperature at $60^{\circ} \mathrm{C}$. Also, the surface morphological of PVA-g-GMA freeze-dried gels was found more compacted, smooth and uniform due to the grafting process. A significant thermal stability was noticed, due to grafting reaction of PVA-g-GMA throughout TGA and DSC results. The obtained results are very promising and opening new area for conducting further investigations considering the very low price of the used UV crosslinking method compared to the chemical crosslinking method.
\end{abstract}

\section{INTRODUCTION}

Hydrogels networks absorb high amount of water and kept water-insoluble, owing to the chemical or physical crosslinking among the polymer network chains (Bhattarai et al., 2010). Over sixty years, hydrogels have been used in numerous biomedical disciplines, in ophthalmology as contact lenses and in surgery fields. These hydrogels facilitate the localized and sustained drug release as they have muco-adhesive and bio-adhesive characteris-

${ }^{*}$ Corresponding Author

Elbadawy A. Kamoun, Polymeric Materials Research Dep., Advanced Technology and New Materials Research Institute, City for Scientific Research and Technological Applications (SRTA-City), New Borg Al-Arab 21934, Alexandria, Egypt.

E-mail:badawykamoun@yahoo.com,e-b.kamoun@tu-bs.de tics that improve drug stability time and tissue permeability and can make them excellent drug delivery vehicles (Bhattarai et al., 2010 and Hamidi et al., 2008). Gelation process can take place by either chemical or physical crosslinking (association, aggregation, crystallization, complexation, and hydrogen bonding) that are reversible due to the conformational changes. While the chemical covalent crosslinking is permanent and irreversible as a result of configurationally changes (Tsung and Burgess, 2012). However, chemical crosslinked gels are mechanically stable, and the rate of biodegradation of the polymer network is generally controllable. Both photo induced and thermal initiation have been used to prepare synthetic covalent hydrogels. Among these methods, photocrosslinking has several advantages such as; (1) it allows better spatial and temporal control over the reaction, (2) chemical solvent-free, (3) relatively low cost, and (4) rapid cell compatibility and entrapment with high cell viability due to the fast curing rates. 
Accordingly, photocrosslinking method is preferred for the preparation of covalent hydrogels especially for biomedical applications (Zhong et al., 2010). PVA is regarded as one of the most frequent and the oldest synthetic hydrophilic polymer, due to its good biocompatibility applied in several advanced biomedical applications e.g. wound dressing (Kamoun et al., 2015a; Kamoun et al., 2015b and Kenawy et al., 2014) wound management (Zhao et al., 2003), drug delivery systems (Kamoun and Menzel, 2010 and Kamoun and Menzel, 2012), artificial organs, and contact lenses (Marin et al., 2014). In addition, PVA is also used in wide application sectors including foods, lacquers, resins and cosmetics industries. In the pharmaceutical field, PVA acts as drug coating agent and as a proper material for surgical sutures (Zhang et al., 2007). It is a semi-crystalline synthetic polymer, not mutagenic, making it suitable for pharmaceutical and biomedical applications (Kamoun et al., 2015a). PVA has been chemically modified with acrylates and methacrylates by various ways. The addition of unsaturations to polymers allows the reticulation of modified polymers with no crosslinkers addition. The reaction might be carried out through the radical initiator pathway i.e. by UV-light. The esterification reaction occurred previously by the reaction between hydroxyl groups of PVA and GMA for modifying PVA (Crispim et al., 2012; Martens and Anseth, 2000 and Zhao et al., 2010). Chemical modification of hydroxylated compounds by the insertion of GMA and its posterior crosslinking has been accomplished as an alternative hydrogel synthesis pathway. For example, Martens and Anseth have used an excess of glycidyl acrylate in aqueous media $\sim \mathrm{pH}$ 1.5 to modify the PVA (Martens and Anseth, 2000). They suggested that the reaction occurred through the opening of the GMA epoxy ring (Martens and Anseth, 2000). Van Dijk-Wolthuis et al., 1995 and Lo \& Jiang, 2010, have investigated the chemical modification of dextran with GMA in DMSO as a solvent catalyzed by 4-( $N, N$-dimethylamino) pyridine (DMAP). They concluded that the addition of GMA to dextran might occur through the opening of the GMA epoxy ring, too. They found glycidol as a by-product, which resulted in the addition of methacrylate groups (Van Dijk-Wolthuis et al., 1995 and Lo \& Jiang, 2010). However, it was postulated that the trans-esterification reaction would be mainly favored because the formed glycidol is a good leaving group (Van Dijk-Wolthuis et al., 1995). Recently, GMA was used to modify alginate for producing thermal in-situ polymerizable hydrogels at physiological temperature for cell encapsulation (Wang et al., 2015). The main goal behind this work is to graft GMA onto PVA and optimize the grafting reaction through the grafting conditions such as, GMA concentration and reaction temperature/time. This work also explores the photocrosslinking of PVA-g-GMA hydrogels using UV-light induced for biomedical applications.

\section{EXPERIMENTAL AND INSTRUMENTAL MEASURE- MENTS}

\section{Materials}

Polyvinyl alcohol (PVA, M $_{\text {wt. }} 72000 \mathrm{~g} / \mathrm{mol}$ ) was obtained from (Merck Schuchardt OHG, Hohenbrunn, Germany). Glycidyl methacrylate (GMA) was obtained from (Sigma-Aldrich, Steinheim, Germany). $N, N, N^{\prime}, N^{\prime}$-Tetramethyl-ethylenediamine (TEMED) was obtained from (ACROS-Organics Fisher Scientific UK LTD, Loughborogh, UK). Irgacure 2959, I ${ }_{2959}$ (2-hydroxy-1- [4-(hydroxyethoxy) phenyl]-2-methyl-1-propanone) was obtained from (Ciba-Chemicals, Basel, Switzerland), and dimethylsulfoxide (DMSO) was purchased from (MSDS-Acros-organics, Steinheim, Germany). The PVA-g-GMA macro-monomer/ I $_{2959}$ mixture solution was photo-cured using UV-lamp ( 6 Watt, at $365 \mathrm{~nm}, 0.08$ Amps): (Model-Upland, CA, USA).

\section{Grafting reaction of PVA-g-GMA macro-monomer}

The used procedure of grafting reaction of GMA onto PVA was first published by Crispim et al. (2006), where this procedure has been herein used with a slight modification. Briefly, PVA was dissolved in DMSO to obtain a $5 \%(\mathrm{w} / \mathrm{v})$ solution. A required amount of GMA was added to PVA solution to obtain the following molar ratios of (PVA/GMA) equal to $(1 / 0.025)$, $(1 / 0.05),(1 / 0.07),(1 / 0.09),(1 / 0.15)$ and $(1 / 0.25)$. TEMED was added as a catalyst in $1.0 \mathrm{~mol} \%$, based on $-\mathrm{OH}$ groups of PVA. The solution was kept under 'gentle stirring for $24 \mathrm{~h}$ at $60^{\circ} \mathrm{C}$. The modified polymers (PVA-GMA) were precipitated and collected in acetone to remove the excess of GMA. The resultant amounts of PVA-g-GMA are washed twice with hot acetone. Figure 1 shows schematic diagram of grafting reaction of PVA with GMA through trans-esterification reaction.

\section{Preparation of PVA-g-GMA hydrogel}

A $20 \%(w / v)$ of PVA-g-GMA macro-monomer was partly re-dissolved in mixture of DMSO and hot distilled water till getting a clear solution, followed by addition of $0.1(\mathrm{w} / \mathrm{v}, \%)$ of $\mathrm{I}_{2059}$ photoinitiator at room temperature. The polymer/photoinitiator mixture solution is kept under stirring away from light to avoid any premature crosslinking, until photocrosslinking step. The solution is then exposed direct to UV-lamp at $0 \mathrm{~cm}$ distance for 30 seconds to obtain crosslinked PVA-g-GMA hydrogel, as shown in Figure 1.

\section{Physicochemical Characterizations}

The grafting efficiency $(\mathrm{GE} \%)$ and grafting percentage (GP\%) of the obtained PVA-g-GMA grafted polymer were calculated depending on the following equations as follows:

$$
\begin{aligned}
& \mathrm{GP}(\%)=\left(\mathrm{W}_{1}-\mathrm{W}_{0}\right) / \mathrm{W}_{0} \times 100 . \\
& \mathrm{GE}(\%)=\left(\mathrm{W}_{\mathrm{PVA}-\mathrm{g}-\mathrm{GMA}}-\mathrm{W}_{\mathrm{PVA}}\right) / \mathrm{W}_{\mathrm{GMA}} \times 100 . \\
& \text { Yield }(\%)=\left[\mathrm{W}_{0} / \mathrm{W}_{\text {solid content }}\right] \times 100 .
\end{aligned}
$$

Where $\mathrm{W}_{1}$ is the weight of graft crosslinked hydrogels (PVA-MA) and $\mathrm{W}_{0}$ is the weight of native polymer (PVA) for GP\%. $\mathrm{W}_{\mathrm{PVA}-\mathrm{g}-\mathrm{GMA}}$ is the weight of obtained vacuum-dried PVA-g-GMA macromonomer, $\mathrm{W}_{\mathrm{PVA}}$ is the initial weight of neat $\mathrm{PVA}$, and $\mathrm{W}_{\mathrm{GMA}}$ is the initial weight of GMA that has been introduced in the grafting reaction (Mohy Eldin et al. 2015). While, $\mathrm{W}_{0}$ is the dried gel weight and $\mathrm{W}_{\text {solid content }}$ is the weight of initial used polymer in case of yield\%. Swelling experiments were performed by immersing the formed hydrogel in distilled water at room temperature till equilibrium swollen polymer occurs and measuring their weight again at interval times for $72 \mathrm{~h}$. The swollen hydrogels were then removed from water, dried carefully with filter paper and weighted. The hydrogels were collected and maintained in vacuum oven for 4 days until constant weight (Mohy Eldin et al. 2015). The equilibrium swelling 
degree at different interval times was calculated from the following equation (Kenawy et al., 2014):
$\operatorname{ESD}(\%)=\left(\mathrm{W}_{\mathrm{s}}-\mathrm{W}_{\mathrm{d}}\right) / \mathrm{W}_{\mathrm{d}} \times 100$

Where; $\mathrm{W}_{\mathrm{s}}$ is the weight of swollen hydrogel samples at interval times and $\mathrm{W}_{\mathrm{d}}$ is the weight of dried ones.

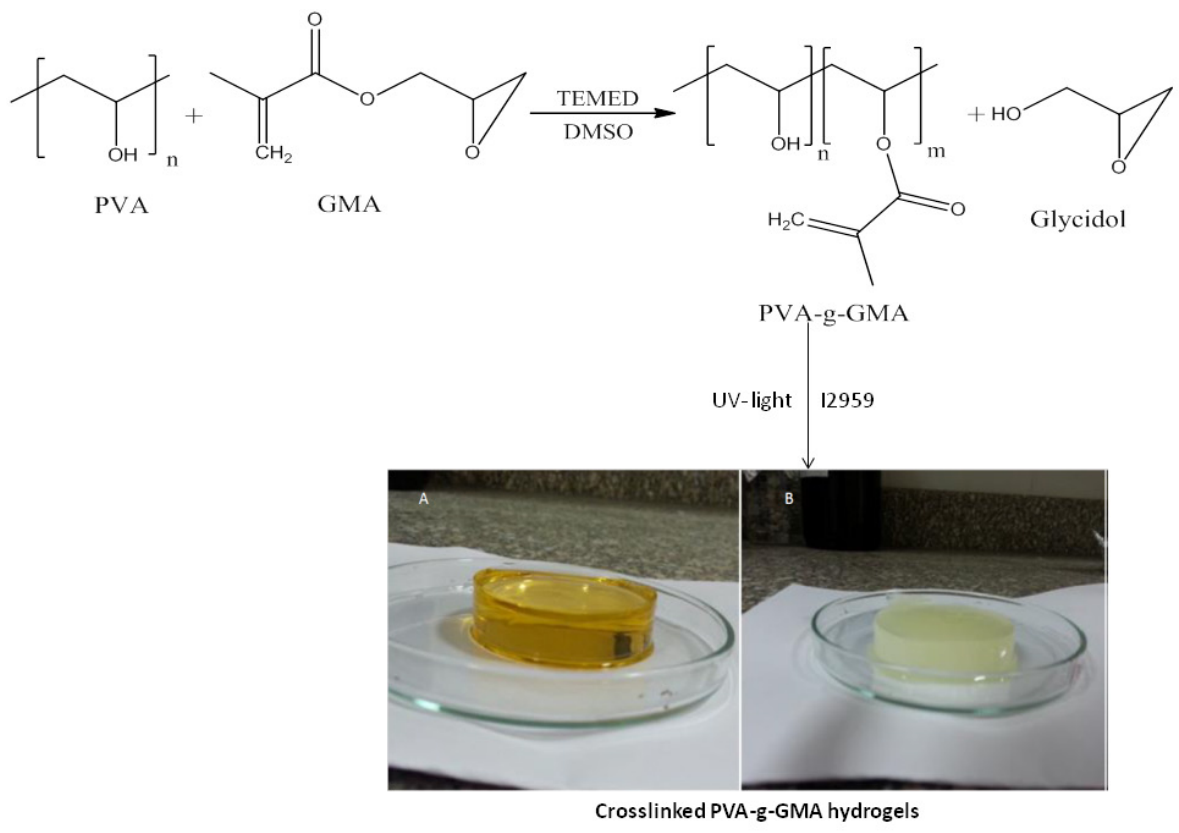

Fig. 1: Schematic diagram showing PVA-g-GMA crosslinked hydrogel formation including trans-esterification grafting reaction of PVA and GMA in DMSO catalyzed by TEMED, followed by UV photo-curing process. (A) Low grafting crosslinked polymer shows a transparent and flexible formed hydrogel, (B) high grafting crosslinked polymer shows an opaque formed hydrogel.

\section{Instrumental Characterizations}

${ }^{1} \mathbf{H}-\mathbf{N M R}$, the proton nuclear magnetic resonance-spectra were recorded by a NMR-DRX400 instrument with $300 \mathrm{MHz}$ (BRUCKER, Karlsruhe, Germany). Typically, $50 \mathrm{mg}$ of the polymeric sample was dissolved in $1.0 \mathrm{~mL}$ of $\mathrm{D}_{2} \mathrm{O}$ or DMSO- $\mathrm{d}_{6}$. Chemical structure of PVA-g-GMA was determined by FTIR spectra for verifying the grafting reaction, (FTIR type: Shimadzu FTIR- 8400 $\mathrm{S}$, Kyoto, Japan). Transparent KBr-disks were prepared by grinding the dried sample with infrared grade $\mathrm{KBr}$ and then pressing. The spectra were detected by recording 64 scans between $4000-400 \mathrm{~cm}^{-1}$ with a resolution of $2 \mathrm{~cm}$. All samples were freeze-dried using liquid nitrogen, crushed to a fine powder (KBr/sample 130/2 mg) respectively, and pressed by applying a force $105 \mathrm{~N}$ into transparent disk (maximum disk weight $\sim 150 \mathrm{mg}$ ) with a diameter $13 \mathrm{~mm}$. All samples were measured in transmittance (\%) mode.

The surface morphology of the freeze-dried PVA-g-GMA gel samples were investigated by an environmental analytical- scanning electron microscope (SEM type: JEOL, JSM-6360LA, Tokyo, Japan) with $15 \mathrm{KV}$ voltage. The hydrogel was first soaked in deionized water for $24 \mathrm{~h}$ to bulge the internal channels and remove any impurities as well. The samples were then dehydrated by a sudden freezing using liquid nitrogen followed by lyophilization at $-90^{\circ} \mathrm{C}$

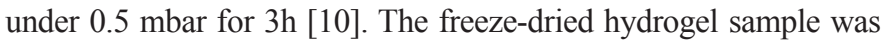
then coated with Au using an ion sputter coater in (model: 11430, USA, connected with vacuum base unit or SPi module control, model: 11425, USA).

The influence of grafting reaction on the thermal stability of PVA-g-GMA crosslinked hydrogels was determined by thermo- gravimetric analysis (TGA) and differential scanning calorimetry (DSC). Both combined thermal analysis instruments of DSC and TGA thermo-grams were recorded by NETZSCH-204 (Phoenix TGA instrument, Selb, Germany). The thermal decomposition was investigated from $50-600^{\circ} \mathrm{C}$ at a heating rate of $10^{\circ} \mathrm{C} / \mathrm{min}$ under $\mathrm{N}_{2}$ flowing rate of $20 \mathrm{~mL} / \mathrm{min}$. Certain thermal kinetic parameters were determined from TGA results, such as $\mathrm{T}_{\text {onset }}$ is defined as the temperature at the intersection of the baseline mass and tangent drawn to the mass curve at the inflection point or point of the greatest rate of mass $\operatorname{loss} \%$. $\mathrm{T}_{\mathrm{d}}$ is the degradation temperature which can be determined at the beginning of the second sharp degradation curve region until the third degradation curve baseline. $\mathrm{T}_{50}$ is defined as the temperature at which the tested sample is thermally degraded and reached $50 \%$ mass loss (Mohy Eldin et al., 2015). While, Glassy transition temperatures $\left(\mathrm{T}_{\mathrm{g}}\right)$ is determined from DSC results, the $\mathrm{T}_{\mathrm{g}}$ is determined as mid-point in the thermograms, as measured from the extensions of the per-and post-transition baselines (Mohy Eldin et al., 2015).

\section{RESULTS AND DISCUSSION}

\section{Grafting verification}

\section{${ }^{1} H$-NMR spectra}

${ }^{1} \mathrm{H}-\mathrm{NMR}$ spectra of PVA, GMA, and PVA-g-GMA $(1 / 0.025 \mathrm{M})$ macro-monomer before crosslinking are presented in Figure 2, respectively. ${ }^{1} \mathrm{H}-\mathrm{NMR}$ spectrum of GMA shows a signal peak at $\delta 1.86 \mathrm{ppm}$ corresponding to the methyl group. In addition, two multiplet signals at the range $\delta 2.629-2.638 \mathrm{ppm}$ and $\delta 2.759$ 
$-2.777 \mathrm{ppm}$ were observed, corresponding to two protons of the $-\mathrm{CH}_{2}$ group in the epoxy ring, $\left(\mathrm{H}_{\mathrm{a}}\right)$ and $\left(\mathrm{H}_{\mathrm{b}}\right)$ (Figure 2). A multiplet signal is observed too at $\delta 3.19-3.22 \mathrm{ppm}$, corresponding to the $-\mathrm{CH}$ group. Also at the range $\delta 3.865-3.904 \mathrm{ppm}$ and $\delta 4.42-4.451 \mathrm{ppm}$, two multiple signals are observed, each equivalent to one proton corresponding to the $\mathrm{O}-\mathrm{CH}_{2}$ group. The presence of singlet peak at $\delta 5.60$ and $6.03 \mathrm{ppm}$ is attributed to the vinyl protons of the methacrylate group (Figure 2). The ${ }^{1} \mathrm{H}-\mathrm{NMR}$ spectrum of the modified PVA-g-GMA grafted macro-monomer shows the appearance of $-\mathrm{CH}_{3}$ and vinyl protons signals, which verifies the grafting step by the presence of methacryloyl groups on PVA. After UV-light placement, the signals of at $\delta 5.6$ and $6.03 \mathrm{ppm}$ are going to disappear as the crosslinking occurs through $\mathrm{C}=\mathrm{C}$ bond that has the vinyl protons as aforementioned verified.

\section{FTIR analysis}

FTIR analysis is a helpful tool to prove the occurred grafting reaction between PVA and GMA forming PVA-g-GMA with molar ratio $(1 / 0.025)$ crosslinked hydrogel, where $\mathrm{C}=\mathrm{C}$ bond disappeared after gelation occurring by UV photopolymerization process. The FTIR spectra of PVA, GMA, and PVA-g-GMA are shown in Figure 3. In the PVA spectrum, the characteristic bands can be detected as follows, -OH groups at $3402 \mathrm{~cm}^{-1}$, the stretching of C-O groups at $1091 \mathrm{~cm}^{-1}$, and $\mathrm{C}-\mathrm{H}$ stretching band was detected at 2918 $\mathrm{cm}^{-1}$. In the spectrum of GMA, some characteristic bands can also be assigned to the respective groups as stretching of $\mathrm{C}=\mathrm{O}$ due to methacryloyl groups at $1712 \mathrm{~cm}^{-1}$, out-of-plan bending of the $\mathrm{R}_{2} \mathrm{C}=\mathrm{CH}_{2}$ group at $937 \mathrm{~cm}^{-1}$, and stretching of the $\mathrm{C}-\mathrm{O}$ (ester) group at 1170 $\mathrm{cm}^{-1}$.

Interestingly, characteristic bands of PVA-g-GMA crosslinked hydrogel can be detected, such as the bending of $\mathrm{C}=\mathrm{O}(1630$ $\left.\sim 1720 \mathrm{~cm}^{-1}\right)$ and absence of out-of-plan bending of $\mathrm{R}_{2} \mathrm{C}=\mathrm{CH}_{2}(937$ $\mathrm{cm}^{-1}$ ) which proves that the graft crosslinked hydrogel formation occurred through the $\mathrm{C}=\mathrm{C}$ bond. It should be pointed out that the intensity of $\mathrm{C}=\mathrm{O}$ band is raised by the increase amount of GMA in the feed solution used in the PVA chemical modification.

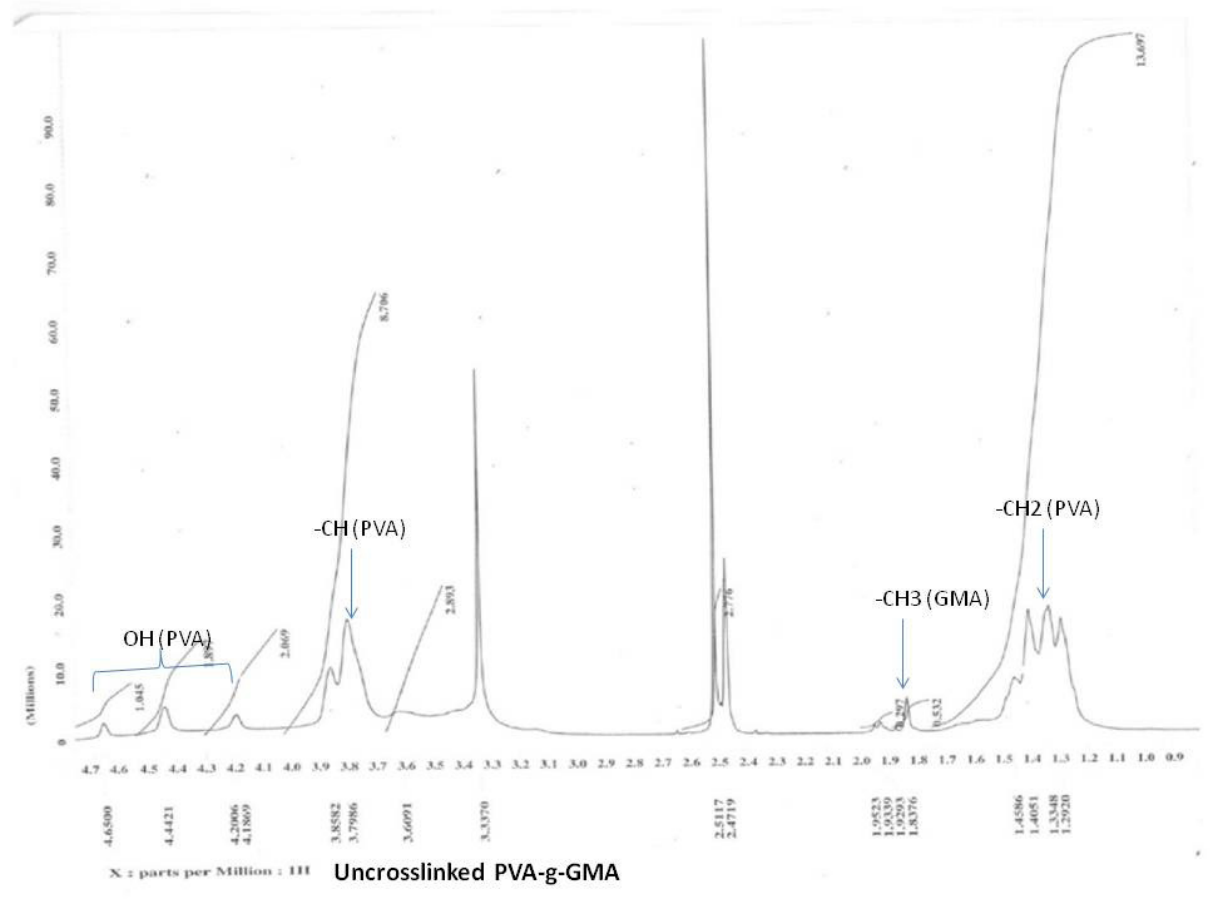

Fig. 2: ${ }^{1} \mathrm{H}-\mathrm{NMR}$ spectrum of PVA-g-GMA macro-monomer with a molar ratio $(1 / 0.025)$.

\section{Morphology investigation by SEM}

The morphology of PVA-g-GMA hydrogels prepared by the strategy of cryo-fixation and cryo-fracturing technique was analyzed by SEM. The morphologies of freeze-dried hydrogels based on PVA-g-GMA synthesized with different ratios of GMA [-OH(PVA)/GMA]: (1/0), (1/0.025), (1/0.05), (1/0.07), (1/0.15), and (1/0.25) $\mathrm{M}$ are presented in Figure 4. As shown, surface morphology of PVA hydrogels (without GMA) presents a rough, rugged and heterogeneous surface structure (Figure 4A), while PVA-g-GMA hydrogels with different GMA ratios exhibit a relatively smooth, uniform surface, compressed, and non-porous surface structure with few tiny cracks which clearly appear by increasing the content of GMA in hydrogels (Figures 4B-F). This might be attributed to increasing amount of GMA onto PVA increases the compactness surface structure of hydrogel owing to the crosslinking density increase, which resulted in a smooth and uniform surface structure appearance. The current influence of grafting reaction of GMA onto PVA on the surface morphology is similar to the influence of grafting L-arginine onto alginate hydrogels and crosslinked dextran-HEMA hydrogels (Mohy Eldin et al., 2015 and Liu et al., 2005). 


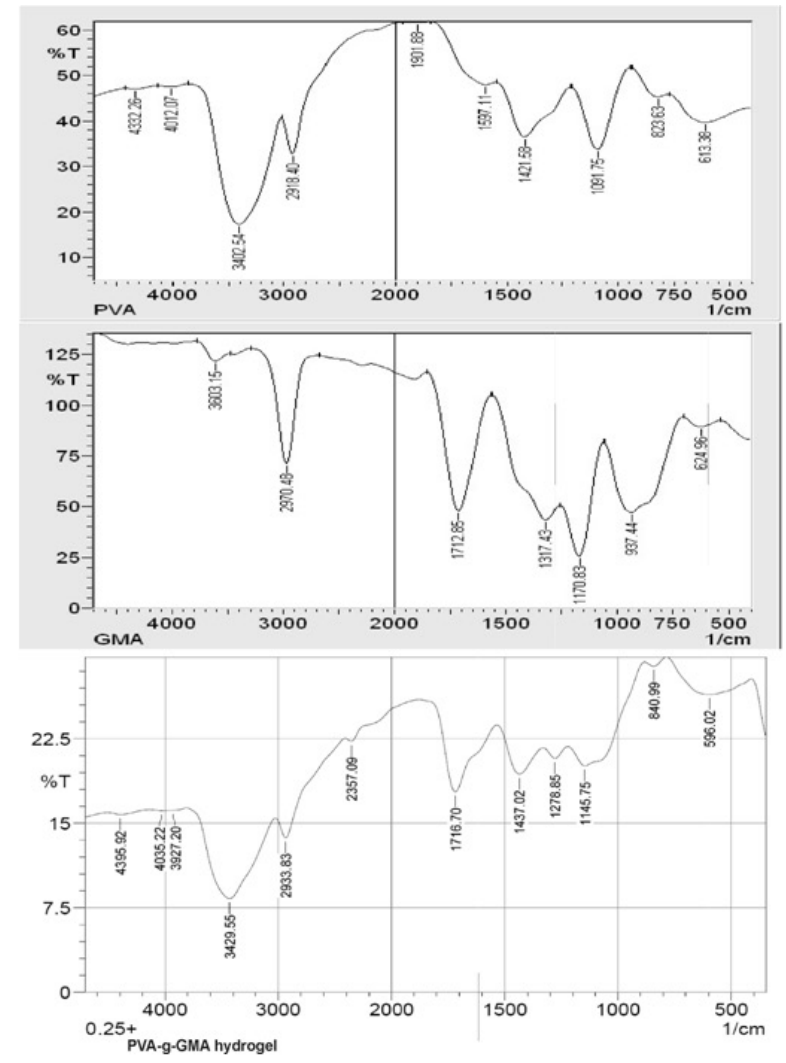

Fig. 3: FTIR spectra of PVA, GMA, and PVA-g-GMA (with molar ratio (1/0.025)) dried hydrogel, respectively.
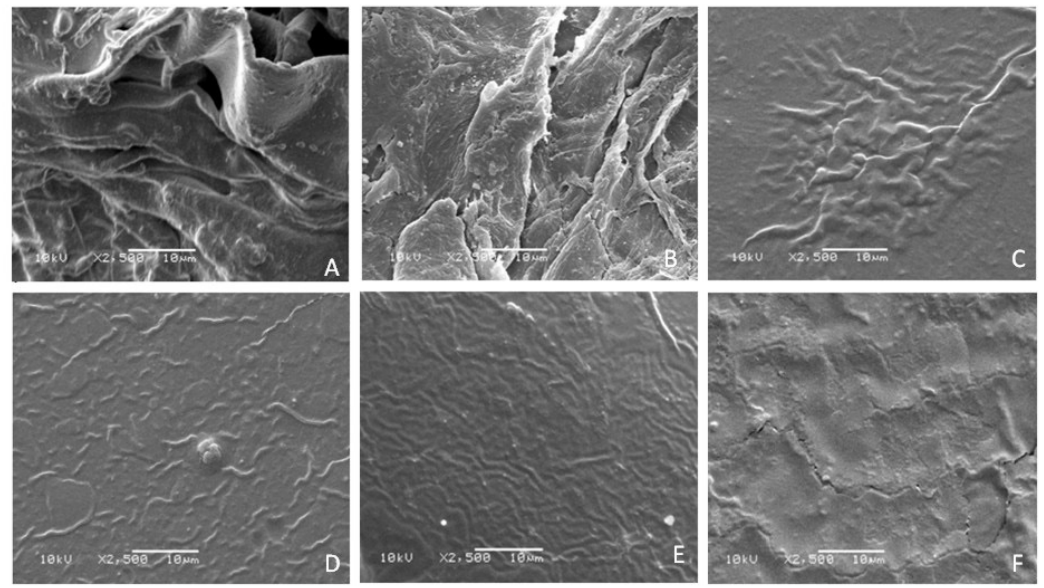

Fig. 4: SEM photographs of crosslinked PVA-g-GMA hydrogels with different GMA ratios as ratios [-OH(PVA)/GMA]: (1/0), (1/0.025), (1/0.05), (1/0.07), (1/0.15), and $(1 / 0.25) \mathrm{M}$ of $(\mathrm{A}, \mathrm{B}, \mathrm{C}, \mathrm{D}, \mathrm{E}$, and F), respectively (original magnification was $\times 2500$ ).

\section{Optimization of grafting reaction}

\section{Effect of GMA concentration}

As GMA is the second main component of hydrogel composition, therefore it was expected that increasing the final weight of the grafted hydrogel over the initial weight of polymer component proves the grafting process. The effect of different GMA concentration [-OH (PVA)/GMA]: (1/0), (1/0.025), (1/0.05), (1/0.07), (1/0.15), and $(1 / 0.25) \mathrm{M}$ at $60^{\circ} \mathrm{C}$ on the grafting percent $(\mathrm{GP} \%)$, and grafting efficiency (GE\%) was exhibited in Figure 5. It is obvious that as the degree of substitution (DS) or grafting degree increases, the grafting percent and efficiency increases. This fact was attributed to the augment in the degree of crosslinking in the hydrogel matrix due to DS rising. Higher DS means high amount of methacrylate groups attached on PVA chains (Crispim et al., 2012), where the initiated sites on PVA consequently increase and a large number of monomer moieties have been attached. The highest GP\% and GE $\%$ were achieved at $77 \%$ and $96 \%$, respectively at [-OH (PVA)/GMA]: (1/0.25 M). 
Table 1. Effect of grafting reaction conditions on yield $\%$ and ESD (\%) of the formed PVA-g-GMA macro-monomer.

\begin{tabular}{cccccc}
\hline GMA ratios, $^{(\mathbf{M})^{\mathbf{a}}}$ & $\begin{array}{c}\text { Yield } \\
(\mathbf{\%})\end{array}$ & $\begin{array}{c}\text { ESD } \\
(\mathbf{\%})\end{array}$ & $\begin{array}{c}\text { Temp. } \\
\left({ }^{\mathbf{}} \mathbf{C}\right)^{\mathbf{b}}\end{array}$ & $\begin{array}{c}\text { Yield } \\
(\mathbf{\%})\end{array}$ & $\begin{array}{c}\text { ESD } \\
(\mathbf{\%})\end{array}$ \\
\hline 0.025 & 89 & 225 & 25 & 55 & 90 \\
0.05 & 92 & 225 & 40 & 90 & 80 \\
0.07 & 95 & 175 & 60 & 96 & 60 \\
0.09 & 96 & 100 & 80 & 98 & 40 \\
0.15 & 97 & 50 & & & \\
0.25 & 98 & 45 & & & \\
\hline
\end{tabular}

a: Grafting reaction was carried out at $60^{\circ} \mathrm{C}$.

b: Grafting reaction was carried out with PVA/GMA molar ratio is $(1 / 0.09 \mathrm{M})$

Table 1 also shows a slow increase in yield $\%$ of grafted methacrylate on PVA from $89 \%$ to $98 \%$ by increasing concentration of GMA. This behavior could be due to the compactness of the gel phase that could hinder the mobility of polymeric chains, thus preventing further crosslinking reactions (Pitarresi et al., 2003). Interestingly, the equilibrium swelling degree (ESD) values have been significantly reduced due to increase GMA ratios. It was due to the formation of a tighter and compacted 3D-network of hydrogel that would reduce the availability of free hydroxyl groups, which correspond to the hydrophilicity of hydrogel. Thus, the penetration of water molecules into the hydrogel network becomes quite difficult, showing a reduction in the swelling degree of the hydrogel. The increase of crosslinking degree restricts the polymeric matrix expansion and then less water amount is absorbed. This result is fully consistent to the result of the grafting and formation of dextran-MA hydrogel (Kim and Chu, 2000). Dextran-methacryalte hydrogels showed a wide range of swelling from $67 \%$ to $227 \%$, where their swelling increases and hydrogel matrices expand with decrease in methacrylate substitutions in hydrogels (Kim and Chu, 2000).

\section{Effect of grafting reaction temperature}

It is well known that as the temperature increases, the rate of reaction increases according to Maxwell-Boltzmann law distribution and Arrhenius kinetic law which is based on an increase in reaction temperature is in general associated with an increase in chemical rate constant. If the substance undergoes heating, the particles move faster and so collide more repeatedly which speeds up the rate of reaction. Thus, increasing the reaction temperature increases the average kinetic energy of its constituent particles which leads the particles to move faster and collide more frequently and possess greater energy when they collide. Figure 6 exhibits the effect of the reaction temperature on the grafting percentage of crosslinked PVA-MA hydrogel, where both GP\% and GE\% increase with rising the reaction temperature up to $60^{\circ} \mathrm{C}$ and then remains constant (Ibrahim et al., 2005). The maximum $\mathrm{GP} \%$ and $\mathrm{GE} \%$ of PVA-MA macro-monomer formed at $60^{\circ} \mathrm{C}$.

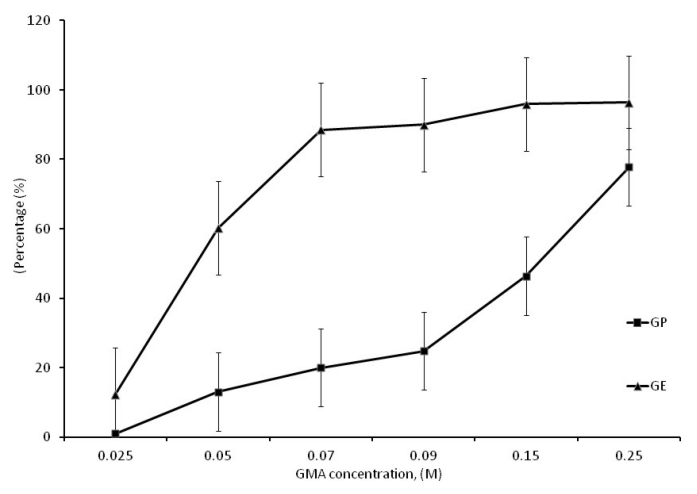

Fig. 5: Effect of various GMA ratios [-OH(PVA)/GMA]: (1/0), (1/0.025), (1/0.05), (1/0.07), (1/0.15), and $(1 / 0.25) \mathrm{M}$ at $60^{\circ} \mathrm{C}$, on the formed PVA-gGMA macro-monomer during the grafting reaction as function of grafting efficiency and percentage.

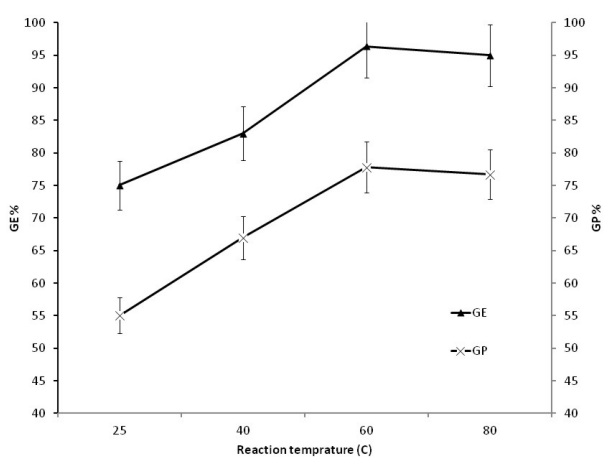

Fig. 6. Effect of different grafting reaction temperatures $\left(25,40,60\right.$, and $\left.80^{\circ} \mathrm{C}\right)$ with defined molar ratio around [-OH(PVA)/GMA]: $(1 / 0.05)$ on the formed PVA-g-GMA macro-monomer during the grafting reaction as function of grafting efficiency and percentage.

It could be explained that increasing reaction temperature results in the reaction of the maximum amount of methacrylate molecules with the hydroxyl groups of PVA chains producing the maximum yield of PVA-g-GMA macro-monomer. This behavior reflects positively on the $\mathrm{GP} \%$ and $\mathrm{GE} \%$ values. The high temperature was helpful in increasing the bimolecular collisions for PVA and GMA that enhances the diffusion of monomer molecules into the matrix. This fact can be attributed to the decrease of solution viscosity due to increasing the grafting temperature which results in high collisions between particles with enough energy, showing further homogeneity and larger amounts of GMA monomer could be easily grafted onto PVA matrix forming PVAg-GMA macro-monomer. Thus, the chance of crosslinking by photopolymerization is increasing too. On the other hand, it was observed a slight change was observed in the grafting behavior after $60^{\circ} \mathrm{C}$. It was attributed to the reaction between GMA and PVA at higher temperature then $60^{\circ} \mathrm{C}$ allowed the possibility of diffusion of all GMA molecules present to the PVA matrix leading to a steric hindrance causing a fast termination reaction (Crispim et al., 2006 and 2012). Table 1 also shows an increase in yield $\%$ of 
grafted methacrylate on PVA by increasing reaction temperature up to $60^{\circ} \mathrm{C}$ which is corresponding to the aforementioned speculation. On the other hand, the ESD\% values have progressively decreased with increasing the grafting temperature until $80^{\circ} \mathrm{C}$. As, the formed hydrogel at $>40^{\circ} \mathrm{C}$ has a tighter and compacted crosslinked network structure which results in short crosslinking chains at high temperature of grafting. Thereby, the diffusion rate of water molecules to the hydrogel network will be very limited, and the swelling degree decreases consequently, (Table 1).

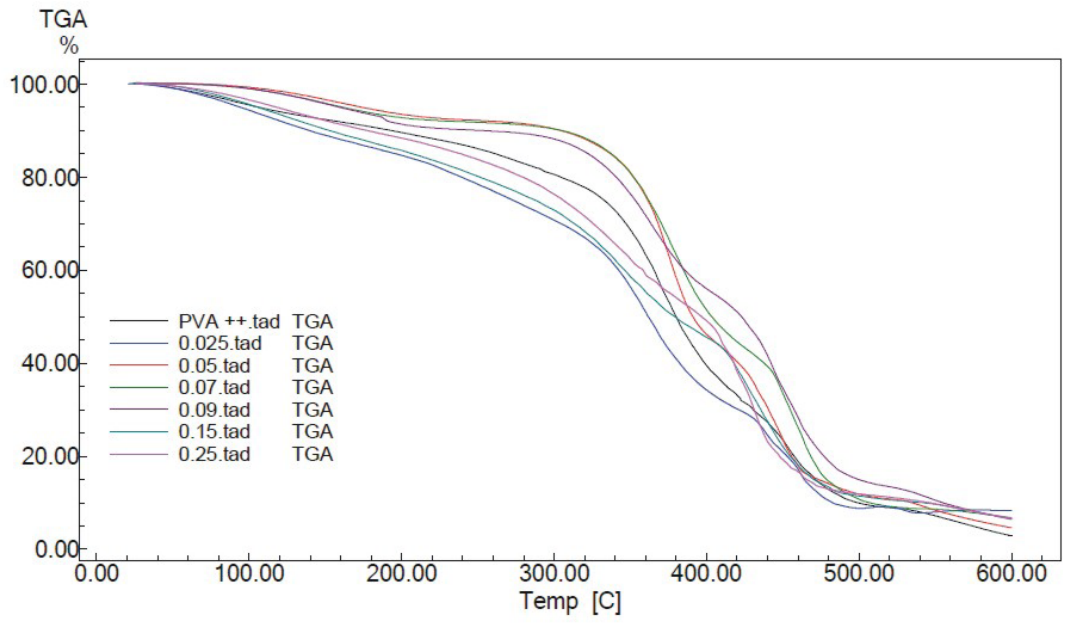

Fig. 7: TGA thermograms curves of PVA-g-GMA crosslinked xerogels with different GMA ratios as presented [-OH(PVA)/GMA]: (1/0), (1/0.025), (1/0.05), (1/0.07), $(1 / 0.15)$, and $(1 / 0.25) \mathrm{M}$, respectively.

Table 2. Thermal properties of PVA-g-GMA xerogels according to the TGA and DSC thermogram results, (the grafting reaction of tested polymers was carried out at $\left.60^{\circ} \mathrm{C}\right)$.

\begin{tabular}{cccccc}
\hline PVA-g-GMA xerogel & DSC results & \multicolumn{2}{c}{ TGA } \\
results
\end{tabular}

\section{Effect of grafting reaction on thermal stability of hydrogels}

Thermal properties of crosslinked neat PVA and PVAg-GMA dried hydrogels have been conducted by TGA and DSC instruments. The thermograms data have been depicted and summarized in Table 2. DSC results show a significant improvement of the thermal stability of PVA-g-GMA, due to the occurrence of grafting reaction. As presented in Table 2, $\mathrm{T}_{\mathrm{g}}$ values increase gradually from $87-184^{\circ} \mathrm{C}$ owing to grafting of GMA onto PVA which in terms increases crosslinking density of hydrogel resulting in satisfied thermal stability until $0.09 \mathrm{M}$ of GMA ratio. Similarly, thermal stability of PVA-g-GMA hydrogels has taken the same thermal behavior according to the presented data of TGA results in Table 2. TGA thermograms curves of PVA-g-GMA hydrogels with different ratios of GMA have been shown in Figure 7 and also summarized in Table 2. It is clearly observed that the $T_{d}$ in the second degradation region, $\mathrm{T}_{\text {onset }}, \mathrm{T}_{50}$, and weight loss of PVAg-GMA hydrogels are enhanced apparently by grafting reaction with GMA, owing to the increase of the crosslinking density in presence of high moieties of GMA fractions in polymer chain. These results are consistent with our previous reported results of grafting of L-arginine onto alginate hydrogel, due to grafting reaction (Mohy Eldin et al., 2015). All TGA determined parameters of PVA-g-GMA hydrogels as aforementioned have ther- 
mally improved due to the increase of the ratio of GMA until 0.09 $\mathrm{M}$, and afterwards they return to reduce again with high ratios of GMA at 0.125 and $0.25 \mathrm{M}$. The thermogravimetric results illustrate that, thermal stability of PVA-g-GMA has been clearly improved by the grafting process. Thus, the highest thermal stability was investigated with the highest grafting percentage (PVA/ GMA of $1 / 0.09 \mathrm{M})$, then thermal stability was deteriorated with reducing the grafting percentage with molar ratios $(1 / 0.125)$ and $(1 / 0.25)$, respectively. This fact was attributed to the amount of grafted methacrylate groups onto PVA improved the thermal stability of PVA-g-GMA, due to their own thermal stability, compared to pristine or low grafted PVA $[20,21]$. Therefore, the ratio of GMA $(0.09 \mathrm{M})$ has been chosen as the optimized ratio for completing the preliminary attempts of PVA-g-GMA hydrogels in the biomedical evaluation assessment which will be explored in the second part of this research.

\section{CONCLUSIONS}

In conclusion, graft polymers of GMA onto PVA using different grafting reaction conditions can be carried out successfully. The proof of grafting reaction was verified from ${ }^{1} \mathrm{H}-\mathrm{NMR}$ and IR analyses. In addition, chemically modified or grafted PVA was photo-cured using photopolymerization technique through UV-light induced. The effect of variation grafting conditions such as; GMA ratios and grafting reaction temperature and subsequently on the grafting efficiency/percentage and yield $\%$ was studied. It was concluded that grafting parameters increases progressively with GMA ratio and also with reaction temperature up to $60^{\circ} \mathrm{C}$. It was worthy to mention here that the grafting reaction of GMA onto PVA has sharply influenced on the surface morphological structure of resultant PVA-g-GMA crosslinked hydrogels, which is owing to the degree of crosslinking density emerging with grafting increase. The study of thermal kinetics of the PVA-g-GMA dried gels prepared with different GMA ratio showed a wide difference comparative with that of the hydrogels prepared without GMA. It was observed that grafting reaction in particular GMA ratio increase has improved the overall thermal stability of UV crosslinked PVA-g-GMA hydrogels until 0.09 $\mathrm{M}$ as GMA ratio, and then hydrogels take an opposite thermal degradation behavior. The results are promising; especially the fact that the cost of the used photocrosslinking of solvent-free would be of interest for biomedical applications such as wound dressings and controlled release systems which will be shown in the second extended part of this paper. The extended second part of this work will be discussed in details parameters affecting on hydrogel formation, swelling behavior and bio-evaluation tests assessment in order to use this prepared hydrogel for biomedical applications.

\section{Conflicts of interest}

The authors report no financial or nonfinancial conflict of interest.

\section{Acknowledgement}

The authors thank Prof. Henning Menzel, Institute for Technical Chemistry, Brunschweig University of Technology, Germany for donating the Irgacure 2959.

\section{REFERENCES}

Bhattarai N, Gunn J, Zhang M. Chitosan-based hydrogels for controlled localized drug delivery. Adv Drug Del Rev, 2010; 62: 83-99.

Crispim EG, Piai JF, Schüquel ITA, Rubira AF, Muniz EC. Functionalization of poly(vinyl alcohol) by addition of methacryloyl groups: Characterization by FTIR and NMR and optimization of reaction conditions by RSM. E-Polymers, 2006; 6: 1-18.

Crispim EG, Piai JF, Fajardo AR. Hydrogels based on chemically modified poly(vinyl alcohol) (PVA-GMA) and PVA-GMA/chondroitin sulfate: Preparation and characterization. Express Polym Lett, 2012; 6: 383-395.

Hamidi M, Azadi A, Rafiei P. Hydrogel nanoparticles in drug delivery. Adv Drug Del Rev, 2008; 60(15): 1638-1649.

Ibrahim NA, Abu-Ilaiwi F, Rahman MZA, Ahmad MB, Dahlan KZM, Yunus WMZW. Graft copolymerization of acrylamide onto Oil Palm Empty Fruit Bunch (OPEFB) fiber. J Polym Res, 2005; 12(3): 173-179.

Kamoun EA, Menzel H. Crosslinking behavior of dextran modified with hydroxyethyl methacrylate upon irradiation with visible lighteffect of concentration, coinitiator type, and solvent. J Appl Polym Sci, 2010; 117(6): 3128-3138.

Kamoun EA, Menzel H. HES-HEMA nanocomposite polymer hydrogel: Swelling behavior and characterization. J Polym Res, 2012; 19: 9851-9865.

Kamoun EA, Chen X, Mohy Eldin MS, Kenawy ES. Crosslinked poly(vinyl alcohol) hydrogels for wound dressing applications: A review of remarkably blended polymers. Arab J Chem, 2015(a); 8(1): 1-14.

Kamoun EA, Kenawy ES, Tamer TM, El-Meligy MA, Mohy Eldin MS. Poly (vinyl alcohol)-alginate physically crosslinked hydrogel membranes for wound dressing applications: Characterization and bio-evaluation. Arab J Chem, 2015(b); 8(1): 38-47.

Kenawy E, Kamoun EA, Mohy Eldin MS, El-Meligy MA. Physically crosslinked poly(vinyl alcohol)-hydroxyethyl starch blend hydrogel membranes: Synthesis and characterization for biomedical applications. Arab J Chem, 2014; 7(3): 372-380.

Kim SH, Chu CC. Synthesis and characterization of dextran-methacrylate hydrogels and structural study by SEM. J Biomed Mater Res, 2000; 49(4): 517-527.

Liu Y, Li Y, Yang L, Liu Y, Bai L. Graft copolymerization of methyl acrylate onto sodium alginate initiated by potassium diperiodatocuprate (III). Iranian Polym J, 2005; 14(5): 457-463.

Lo CW, Jiang H. Photo-patterning and degradation study of dextran-glycidyl methacrylate hydrogels. Polym Eng Sci, 2010; 2010: 232-239.

Marin E, Rojas J, Ciro Y. A review of polyvinyl alcohol derivates: Promising materials for pharmaceutical and biomedical applications. Afr J Pharm Pharmacol, 2014; 8(24): 674-684.

Martens P, Anseth KS. Characterization of hydrogels formed from acrylate modified poly(vinyl alcohol) macromers. Polymer (Guildf), 2000; 41: 7715-7722.

Mohy Eldin MS, Kamoun EA, Sofan MA, Elbayomi SM. L-Arginine grafted alginate hydrogel beads: A novel $\mathrm{pH}$-sensitive system for specific protein delivery. Arab J Chem, 2015; 8: 355-365.

Pitarresi G, Palumbo FS, Giammona G, Casadei M, Micheletti Moracci F. Biodegradable hydrogels obtained by photocrosslinking of dextran and polyaspartamide derivatives. Biomaterials, 2003; 24(23): 43014313.

Tsung J, Burgess DJ. Fundamentals and applications of controlled release drug delivery: Fundamentals and Applications of Controlled Release Drug Delivery. Advances in Delivery Science and Technology; Springer, 2012; 2012: 107-123.

Van Dijk-Wolthuis WNE, Franssen O, Talsma H, van Steenbergen MJ, Kettenes-van den Bosch J J, Hennink WE. Synthesis, characterization and polymerization of glycidyl methacrylate derivatized dextran. Macromolecules, 1995; 28(18): 6317-6322.

Wang X, Hao T, Qu J, Wang C, Chen H. Synthesis of therma polymerizable alginate-GMA hydrogel for cell encapsulation. J Nanomater, 
2015; 2015: 1-8.

Zhang L, Yu P, Luo Y. Dehydration of caprolactam-water mixtures through cross-linked PVA composite pervaporation membranes. J Memb Sci, 2007; 306: 93-102.

Zhao L, Mitomo H, Zhai M, Yoshii F, Nagasawa N, Kume T. Synthesis of antibacterial PVA/CM-chitosan blend hydrogels with electron beam irradiation. Carbohyd Polym, 2003; 53: 439-446.

Zhao L, Xiong Y, Liu M, Qi X. Study on superabsorbent of maleic anhydride/acrylamide semi-interpenetrated with poly(vinyl alcohol). Polym Adv Technol, 2010; 21(7): 483-489.

Zhong C, Wu J, Reinhart-King C, Chu CC. Synthesis, character- ization and cytotoxicity of photo-crosslinked maleic chitosan-polyethylene glycol diacrylate hybrid hydrogels. Acta Biomater, 2010; 6 (10): 3908-3918.

\section{How to cite this article:}

Kamoun EA, Omer AM, Khattab SN, Ahmed HM, Elbardan AA. In-Situ UV-Photopolymerized PVA-g-GMA Hydrogels for Biomedical Applications: I. Synthesis, Characterizations and Grafting Optimization. J App Pharm Sci, 2018; 8 (01): 034-042. 